\section{Aeromonas hydrophila endocarditis with ruptured mycotic aneurysm of right renal artery}

\author{
Mara Elena Pugliese, ${ }^{1}$ Marco Falcone, ${ }^{2}$ \\ Alessandra Oliva, ${ }^{2}$ Federico Faccenna, ${ }^{3}$ \\ Denise D'Aluisio, ${ }^{1}$ Sergio Morelli' \\ 'Department of Internal Medicine, \\ ${ }^{2}$ Department of Public Health and \\ Infectious Diseases, ${ }^{3}$ Department of \\ Vascular Surgery, "La Sapienza” \\ University, Rome, Italy
}

\section{Abstract}

Aeromonas hydrophila has been infrequently reported as a cause of infection in humans. It has been associated with a variety of clinical syndromes but Aeromonas-related endocarditis is extremely rare. We present the case of a 76year-old diabetic patient who was admitted to our hospital due to severe lumbar pain resistant to nonsteroidal anti-inflammatory drugs accompanied by fever (up to $38.5^{\circ} \mathrm{C}$ ). The vital signs were normal and the physical examination was unremarkable except for tenderness over right flank. Laboratory investigation showed a mild leukocytosis (white blood cell count of $11,360 \times 10^{6} / \mathrm{L}$ ) with elevation of inflammatory markers. Cardiac ultrasound showed a large vegetation on the mitral valve. Abdominal computed tomogrpahy revealed a ruptured aneurysm of the right renal artery. Multiple sets of blood culture grew A. hydrophila.

\section{Introduction}

Aeromonas hydrophila is the most wellknown of the twenty-seven species belonging to the genus Aeromonas. It is an ubiquitous gram negative rod, single polar flagellum, oxidase and catalase positive, fermentative bacterium that inhabits aquatic environments. ${ }^{1}$ Some strains of $A$. hydrophila are capable of causing disease in fishes and amphibians as well as in humans who may acquire infections through open wounds or by ingestion of contaminated water or food (produce, dairy, or meats). ${ }^{2}$ A. hydrophila infection is associated with gastroenteritis, and disseminated infections are more likely to occur in immunocompromised hosts. ${ }^{2,3}$

A mycotic aneurysm is defined as a localized, irreversible dilatation of an artery due to the disruption of the vessel wall caused by infection. Mycotic aneurysms secondary to endocarditis generally involve the aorta, as the primary branches of the aorta, the femoral, cerebral and visceral arteries are the next most common sites. ${ }^{4}$

\section{Case Report}

A 76-year-old man was admitted to our hospital because of persistent severe lumbar pain accompanied by fever (up to $38.5^{\circ} \mathrm{C}$ ). He was affected by type- 2 diabetes mellitus, systemic hypertension, and coronary artery disease. The pain was described as sharp and violent, localized in right side lumbar area without radiation. There was no nausea, vomiting, or diarrhea, and the patient denied a history of recent trauma. Neither dysuria nor stranguria were reported. The vital signs were normal and the physical examination was unremarkable except for tenderness over right flank. Laboratory analyses showed white blood cell count of $11,360 \times 10^{6} / \mathrm{L}$, erythrocyte sedimentation rate $56 \mathrm{~mm} / \mathrm{h}, \mathrm{C}$ reactive Protein $16.46 \mathrm{mg} / \mathrm{dL}$ (normal values 0$0.5 \mathrm{mg} / \mathrm{dL}$ ), fibrinogen $623 \mathrm{mg} / \mathrm{dL}$, D-dimer 1069 $\mathrm{ng} / \mathrm{L}$ (normal values <500 ng/L), hyperglycemia $(319 \mathrm{mg} / \mathrm{dL})$.

Lumbosacral X-ray and abdominal ultrasound did not show abnormal findings.

Transthoracic echocardiogram revealed a large vegetation $(1 \mathrm{~cm})$ on the tip of the anterior leaflet of mitral valve. No hemodynamically significant regurgitation was found. Three sets of blood cultures were collected after admission and incubated at $37^{\circ} \mathrm{C}$. After $48 \mathrm{~h}$, a non-motile, non-fermenter, oxidase and catalase positive Gram negative bacillus grew on blood agar plates, which was further identified as A. hydrophila throughout both VITEK-2 system (Bio-Merieux, Marcy l'Etoile, France) and Matrix-Assisted Laser Desorption/Ionization Time of Flight Mass Spectrometry (MALDI-TOF MS). Antimicrobial susceptibility testing was performed by the VITEK-2 system. The isolate was susceptible to amikacin, cefepime, ceftriaxone, cefotaxime, ceftazidime, ciprofloxacin, ertapenem, gentamicin, intermediate to imipenem and meropenem, and resistant to amoxicillin/ clavulanic acid. Antibiotic therapy with gentamicin at dosage of $3 \mathrm{mg} / \mathrm{kg} /$ die plus ceftriaxone at dosage of $2 \mathrm{~g} /$ die was started. Abdominal CT angiography showed a right renal artery aneurysm $(30 \times 35 \times 35 \mathrm{~mm})$ (Figure 1). Entire right kidney was non-perfused. The aneurysm had ruptured just downstream the aorto-renal junction and was surrounded by a hematoma that involved peri-aortic fatty tissue and right diaphrag pilaster. A Zenith Cook aortic endograft was introduced just below the origin of the superior mesenteric artery, and a Viabahn stent-graft with periscope technique was positioned to maintain perfusion of the left renal artery. Color Doppler ultrasonography was performed 1, 3 and 6 months after the vascular intervention and showed patency of both left renal artery
Correspondence: Maria Elena Pugliese, Department of Internal Medicine, "La Sapienza" University, Viale del Policlinico 155, 00161 Rome, Italy.

Fax: +39.06.499.70211.

E-mail: mariaelena.pugliese@uniroma1.it

Key words: Infective endocarditis; Aeromonas hydrophila; Mycotic aneurysm.

Contributions: the authors contributed equally.

Received for publication: 21 March 2016.

Revision received: 19 July 2016.

Accepted for publication: 26 July 2016 .

This work is licensed under a Creative Commons Attribution-NonCommercial 4.0 International License (CC BY-NC 4.0).

(C) Copyright M.E. Pugliese et al., 2016

Licensee PAGEPress, Italy

Infectious Disease Reports 2016; 8:6515

doi:10.4081/idr.2016.6515

and abdominal aorta. At 6-month follow-up the patient had a near-normal renal function.

\section{Discussion}

The genus Aeromonas is responsible worldwide for a broad number of intestinal and extraintestinal diseases and syndromes. ${ }^{5,6}$ The main species considered to be pathogenic in humans are A. hydrophila, A.s caviae and A. veronii biotype sobri. ${ }^{7}$ The most common clinical manifestation is gastrointestinal infection, although Aeromonas has been associated with a variety of clinical syndromes, including necrosis of muscle, soft tissue and skin, endocarditis, meningitis following craniotomy, infections of surgical and nonsurgical wounds, chronic otitis media, lower respiratory tract infection, and cellulitis. ${ }^{2}$ Disseminated infections are more likely to occur in immunocompromised hosts and Aeromonas-related endocarditis is extremely rare, with only five cases in the world literature. Multiple chronic and/or immunocompromising diseases underlie these few cases: cirrhosis and renal failure; ${ }^{8}$ chronic hepatitis-B infection; ${ }^{9}$ Human Immunodeficiency virus and hepatitis- $\mathrm{C}$ virus co-infection in intravenous drug user patient; ${ }^{10}$ myelodysplastic syndrome; transfusion-related non-A, non-B hepatitis with cirrhosis; colon carcinoma in situ, and subacute thyroiditis. ${ }^{11}$

In this patient, no particular predisposing condition for invasive infections from Aeromonas spp. was found, suggesting the possibility of septicemia with life-threatening complications even in immunocompetent subjects. Another peculiar aspect of this case is that infective endocarditis was complicated by 
a renal mycotic aneurysm (MAs). MAs occur in $2-10 \%$ of infective endocarditis cases and usually involve the aorta; the primary branches of the aorta, the femoral, cerebral and visceral arteries are the next most common sites..$^{412}$ The infection usually invades the vessel wall
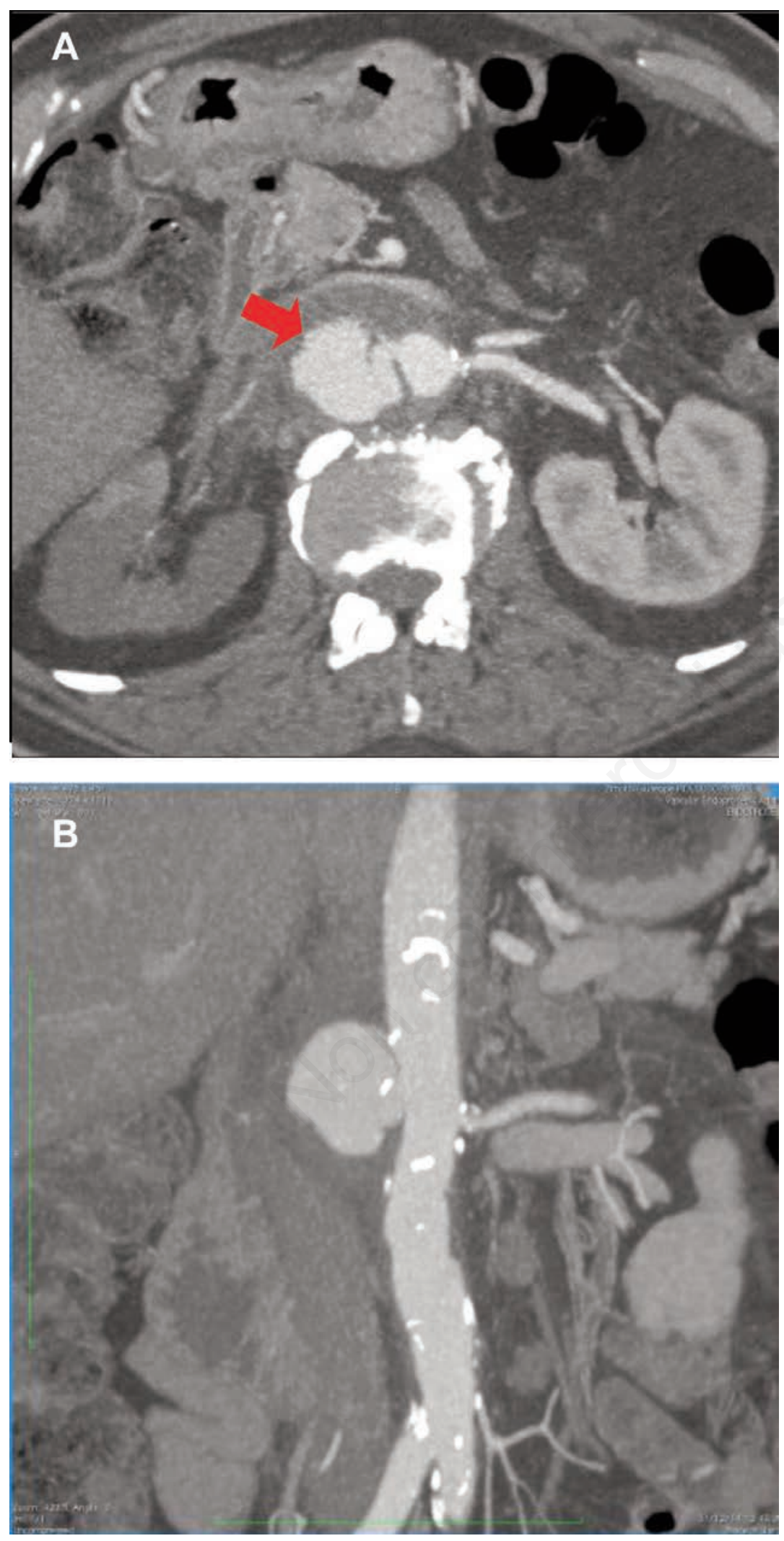

Figure 1. A) Contrast-enhanced computed tomography of abdomen, transverse section: $3 \times 3.5 \times 3.5 \mathrm{~cm}$ aneurysmal vascular structure (arrow) at the right proximal renal artery. B) Contrast-enhanced computed tomography of abdomen, coronal section. through a diseased intima (most commonly by atherosclerosis), or through vasa vasorum. ${ }^{13}$ Apart from sepsis, patients with infectious aneurysms are endangered by imminent rupture of the aneurysm due to accelerated destruction of the vessel wall. ${ }^{14}$ Mycotic aneurysms of renal arteries have rarely been described, ${ }^{15-17}$ and rupture is often associated with life-threatening hemorrhages. ${ }^{18}$ With regard to the antimicrobial therapy, although fluoroquinolones have been reported as the first choice treatment for Aeromonas infections, microorganisms resistant to nalidixic acid and susceptible to ciprofloxacin are known to already have a mutation in GyrA gene that can easily develop a second mutation of resistance, generating a MIC of ciprofloxacine above the breakpoint..$^{19}$ In vitro antimicrobial susceptibility of clinical isolates of Aromonas hydrophila showed resistance to ampicillin, a common trait of Aeromonas spp. strains, and high susceptibility to piperacillin/tazobactam, aminoglycosides and cephalosporins, except cefazolin. A detailed knowledge of the antimicrobial susceptibility patterns was of paramount importance in order to implement the appropriate antimicrobial therapy. ${ }^{20}$ Thus, we observed a good response to antibiotic treatment including gentamicin plus ceftriaxone administered for 4 weeks, and despite the large size of vegetation it was possible to cure the patient without surgical valvular replacement.

\section{Conclusions}

We reported a very uncommon case of infective endocarditis caused by Aeromonas hydrophila complicated by a mycotic aneurysm of the right renal artery in a patient without any classic risk factors for this infection. The patient completely recovered with antibiotic therapy with a $3^{\text {rd }}$ generation cephalosporin plus an aminoglycoside for 4 weeks.

\section{References}

1. Jorgensen JH, Pfaller MA. Manual of clinical microbiology. 11 ed. Washington DC: ASM Press; 2015.

2. Janda JM, Abbott SL. The genus Aeromonas: taxonomy, pathogenicity, and infection. Clin Microbiol Rev 2010;23:35-73.

3. Mukhopadhyay C, Chawla K, Sharma Y, Bairy I. Emerging extra-intestinal infections with Aeromonas hydrophila in coastal region of southern Karnataka. J Postgrad Med 2008;54:199-202.

4. Guzzetta PC. Congenital and acquired aneurysmal disease. Semin Pediatr Surg 1994;3:97-102. 
5. Vila J, Ruiz J, Gallardo J, et al. Aeromonas spp. and traveler's diarrhea: clinical features and antimicrobial resistance. Emerg Infect Dis 2003;9:552-5.

6. Janda JM, Abbott SL. Human pathogens. In: Austin B, Altwegg M, Gosling PJ, Joseph S, (eds.) The genus Aeromonas. West Sussex: John Wiley \& Sons Ltd; 1996. pp 151-173.

7. Janda JM, Abbott SL, Morris JG Jr, eds. Aeromonas, Plesiomonas, and Edwardsiella. In: Infections of the gastrointestinal tract. New York: Raven Press; 1995. pp 905-917.

8. Davis WA, Kane JG, Gargusi VF. Human aeromonas infections: a review of the literature and a case report of endocarditis. Medicine 1978; 57:267-77.

9. Cheong TH, Wang YT, Poh SC. Aeromonas endocarditis in a patient with chronic hepatitis-B infection. Singapore Med J 1989;30:490-2.

10. Hung TW, Lee YT, Chuang ML, et al. Aeromonas endocarditis in an intravenous drug user patient with human immunodeficiency virus and hepatitis $\mathrm{C}$ virus coinfection: a case report. J Emerg Crit Care Med 2012;23:144-51.

11. Ong KR, Sordillo E, Frankel E. Unusual case of Aeromonas hydrophila endocarditis. J Clin Microbiol 1991;29:1056-7.

12. McCready RA, Bryant MA, Divelbiss JL, et al. Arterial infections in the new millennium: an old problem revisited. Ann Vasc Surg 2006;20:590-5.

13. Anderson CB, Butcher HR Jr, Ballinger WF. Mycotic aneurysms. Arch Surg 1974;109: 712-7.

14. Shinonaga M, Kanazawa H, Nakazawa S, et al. Rupture of a smoldering mycotic aneurysm of the thoracic aorta into the lung. Ann Thorac Cardiovasc Surg 2002;8: 177-79.

15. Tardy FG, Vahdat N, Herts BR. Bilateral mycotic renal artery aneurysms. J Urol 2013;189:1908-9.

16. Chao CM, Lai CCJ. Renal artery mycotic aneurysm in a drug abuser. Emerg Med 2013;45:133-4.

17. Marty-Ané $\mathrm{C}$, Alric $\mathrm{P}$, Prudhomme $\mathrm{M}$, et al. Bilateral splenorenal bypass and axillofemoral graft for management of juxtarenal mycotic aneurysm. Cardiovasc Surg 1996;4:331-4.

18. Bayer AS, Bolger AF, Taubert KA, et al. Diagnosis and management of infective endocarditis and complications. Circulation 1998;98:2936-48.

19. Ruiz J, Gómez J, Navia MM, et al. High prevalence of nalidixic acid resistant, ciprofloxacin susceptible phenotype among clinical isolates of Escherichia coli and other Enterobacteriaceae. Diagn Microbiol Infect Dis 2002;42:257-61.

20. Vila J, Marco F, Soler L, et al. In vitro antimicrobial susceptibility of clinical isolates of Aeromonas caviae, Aeromonas hydrophila, and Aeromonas veronii biotype sobria. J Antimicrob Chemother 2002;49:697-702. 